Submitted to the Astrophysical Journal

\title{
Waiting in the Wings: Reflected X-ray Emission from the Homunculus Nebula
}

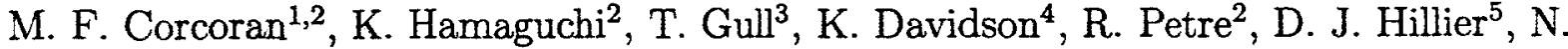

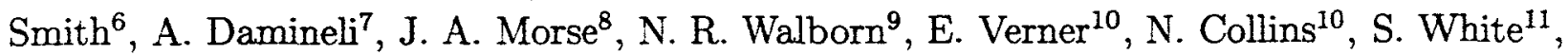 \\ J. M. Pittard ${ }^{12}$, K. Weis ${ }^{13}$, D. Bomans ${ }^{13}$, Y. Butt ${ }^{14}$
}

\footnotetext{
ABSTRACT

${ }^{1}$ Universities Space Research Association, 7501 Forbes Blvd, Ste 206, Seabrook, MD 20706

${ }^{2}$ Laboratory for High Energy Astrophysics, Goddard Space Flight Center, Greenbelt MD 20771; corcoran@lheapop.gsfc.nasa.gov; kenji@lheapop.gsfc.nasa.gov

${ }^{3}$ Laboratory for Astronomy and Solar Physics, NASA/Goddard Space Flight Center, Code 681, Greenbelt, MD 20771 USA; gull@sea.gsfc.nasa.gov

${ }^{4}$ Astronomy Department, University of Minnesota, 116 Church St. SE, Minneapolis, MN 55455 US; kd@astro.umn.edu

${ }^{5}$ Dept. of Physics and Astronomy, University of Pittsburgh, 3941 O'Hara St., Pittsburgh, PA 15260 USA; JDH@galah.phyast.pitt.edu

${ }^{6}$ Center for Astrophysics and Space Astronomy, University of Colorado, 389 UCB, Boulder, CO 80309 USA; nathans@ozma.colorado.edu

${ }^{7}$ Instituto de Astronomia, Geofísica e Ciências Atmosféricas, Universidade de São Paulo, Rua do Matão 1226, 05508-900 São Paulo, SP, Brazil; damineli@astro.iag.usp.br

${ }^{8}$ Arizona State University, Department of Physics \& Astronomy, Box 871504, Tempe, AZ, 85287-1504; jon.Morse@asu.edu

${ }^{9}$ Space Telescope Science Institute, 3700 San Martin Drive, Baltimore, MD 21218 USA; walborn@stsci.edu

${ }^{10}$ IACS/Department of Physics, Catholic University of America, 620 Michigan Ave., NE Washington, DC 20064; kverner@fe2.gsfc.nasa.gov; collins@zolo.gsfc.nasa.gov

${ }^{11}$ Department of Astronomy, University of Maryland, College Park, MD 20742 USA; white@astro.umd.edu

${ }^{12}$ School of Physics and Astronomy, The University of Leeds, Woodhouse Lane, Leeds, LS2 9JT, UK; jmp@ast.leeds.ac.uk

${ }^{13}$ Astronomisches Institut, Ruhr-Universiẗ̈at Bochum, Universitätsstr. 150, 44780 Bochum, Germany; kweis@astro.rub.de; bomans@astro.ruhr-uni-bochum.de

${ }^{14}$ Harvard-Smithsonian Center for Astrophysics, 60 Garden Street, Cambridge, MA 02138 USA; ybutt@head-cfa.harvard.edu
} 
We report the first detection of X-ray emission associated with the Homunculus Nebula which surrounds the supermassive star $\eta$ Carinae. The emission is characterized by a temperature in excess of $100 \mathrm{MK}$, and is consistent with scattering of the time-delayed X-ray flux associated with the star. The nebular emission is bright in the northwestern lobe and near the central regions of the Homunculus, and fainter in the southeastern lobe. We also report the detection of an unusually broad Fe K fluorescent line, which may indicate fluorescent scattering off the wind of a companion star or some other high velocity outflow. The $\mathrm{X}$-ray Homunculus is the nearest member of the small class of Galactic X-ray reflection nebulae, and the only one in which both the emitting and reflecting sources are distinguishable.

Subject headings: circumstellar matter - ISM: individual (Homunculus Nebula) — stars: individual ( $\eta$ Carinae) - reflection nebulae $-\mathrm{X}$-rays: stars

\section{Introduction}

The Homunculus (Gaviola 1950) is a young, hollow, expanding bipolar nebula surrounding the extremely luminous and massive star $\eta$ Carinae. It is believed to have a total mass of 2-12M $\odot$ (Smith, Gehrz \& Krautter 1998; Smith et al. 2003a) and was ejected from the star during the "Great Eruption" of $\eta$ Carinae in the 1840s. At about $100 M_{\odot}$ (Davidson \& Humphreys 1982) $\eta$ Carinae is one of the most massive stars known. It possesses an enormous stellar wind, characterized by a mass loss rate of approximately $10^{-4}-10^{-3} M_{\odot} \mathrm{yr}^{-1}$, and a wind velocity of about $500 \mathrm{~km} \mathrm{~s}^{-1}$ (Hillier et al. 2001). $\eta$ Carinae is a strong source of hard X-rays (Seward et al. 1979) and it undergoes a deep X-ray minimum every 5.53 years (Ishibashi et al. 1999; Corcoran et al. 2001) that lasts for about 3 months. It has been suggested (Corcoran et al. 1997; Pittard et al. 1998; Ishibashi et al. 1999; Corcoran et al. 2000) that the variable X-ray emission is produced by the collision of $\eta$ Carinae's wind with a less dense, faster moving wind from an otherwise hidden companion (though alternative single-star models have been suggested, Davidson 1999). $\eta$ Carinae began its most recent X-ray minimum on June 29, 2003 (Corcoran 2003) and lasted through September 3, 2003.

A new observation by the CHANDRA X-ray Observatory during $\eta$ Carinae's recent $\mathrm{X}$ ray intensity minimum has for the first time identified X-ray emission from the Homunculus nebula itself. This emission is at least a factor of four fainter than the direct stellar emission detected during the minimum, and about a factor of 100 fainter than the direct stellar emission outside of the minimum. The emission associated with the Homunculus was not seen previously by CHANDRA or other X-ray observatories since it is hidden by the wings 
of the instrumentally broadened central source outside of the X-ray minimum. In this paper we report the spectral, spatial and temporal properties of the X-rays from the Homunculus. A more detailed discussion of these data regarding the $\mathrm{X}$-ray emission from $\eta$ Carinae and its surroundings during the X-ray eclipse interval will be presented elsewhere (Corcoran et al.. 2004a, in preparation).

This paper is organized as follows. The observations are presented in $\S 2$. The X-ray and optical imaging of $\eta$ Carinae and its surroundings are compared in $\S 3$. In $\S 4$ we analyze the X-ray spectrum of the Homunculus, and interpret this emission in $\S 5$. We discuss spatial and temporal variations of the emission in $\S 6$, and present our conclusions in $\S 7$.

\section{Observations}

To help characterize the nature of the X-ray minimum, we planned five observations with the CHANDRA X-ray Observatory before, during and after this event as part of a large, multi-wavelength observing campaign during the summer of 2003. The CHANDRA observations used the Advanced CCD Imaging Spectrometer (ACIS) spectroscopic array (Garmire et al. 2003) plus the High Energy Transmission Gratings (Markert et al. 1994). One observation was obtained on July 20, 2003 (CHANDRA sequence number 200216) when contemporaneous monitoring with the RXTE satellite observatory showed $\eta$ Carinae to be in the middle of its X-ray eclipse ${ }^{1}$. The total exposure time was 90,275 seconds. We re-extracted good "Level 2" events from the "Level 1" events file using the CHANDRA Interactive Analysis of Observations (CIAO) software package and the processing steps recommended by the CHANDRA X-ray Center ${ }^{2}$. We included corrections for charge transfer inefficiency (CTI) and destreaking. The effective resolution of the screened photon events file was increased using the algorithm of Tsunemi et al. (2001) .

A followup director's discretionary time observation with ACIS was obtained on August 28,2003 (sequence number 200237) in order to confirm the detection of X-ray emission from the Homunculus and to look for changes in the extended emission which might have occurred as the central source brightened. This observation was obtained with $\eta$ Carinae placed on the ACIS S3 chip, though to minimize the exposure time required the gratings were not used. In order to mitigate effects from pileup of the central source we used a $1 / 8$ sub-array during

\footnotetext{
${ }^{1}$ http://heawww.gsfc.nasa.gov/users/corcoran/eta_car/etacar_rxte_lightcurve/.

${ }^{2}$ http://cxc.harvard.edu/ciao/threads/data.html

${ }^{3} \mathrm{http}: / / \mathrm{cxc}$.harvard.edu/cont-soft/software/subpixel_resolution.1.4.html
} 
the observation. The total exposure time of this observation was 18,796 seconds. As above, we re-extracted the level 2 photon events and corrected for CTI, destreaking and improved the spatial resolution using the algorithm of Tsunemi et al. (2001).

\section{X-ray and Optical Imaging}

Figure 1 shows the zeroth-order X-ray image of $\eta$ Carinae obtained by the ACIS-S3 CCD. The color coding of the image represents emission from gas at different temperatures: red corresponds to the temperature range 2-15 million degrees $(0.2-1.5 \mathrm{keV})$, green $15-30$ million degrees $(1.5-3.0 \mathrm{keV})$, and blue $30-100$ million degrees $(3-10 \mathrm{keV})$. $\eta$ Carinae is visible as a blue-white source at the center of the image. As seen previously (Seward et al. 2001), an incomplete elliptical ring or shell of soft X-ray emission (which appears red in figure 1) nearly surrounds $\eta$ Carinae at a distance of about 2.2 light years from the star. An elongated patch of bluish (hard X-ray) emission about 17" in length (about 0.6 light years) is clearly visible in Figure 1 between the star and the outer red shell. This hard emission was probably detected in earlier ACIS observations (Seward et al. 2001; Weis, Corcoran, Bomans, \& Davidson 2004), as a halo of scattered X-rays surrounding the central stellar source; however contamination by X-rays from the bright, heavily piled up central source did not allow this halo to be clearly identified. Figure 1 also shows an HST/WFPC2 image of the nebulae surrounding $\eta$ Carinae, at the same scale and orientation as the X-ray image. $\eta$ Carinae is not visible in the image, which is scaled in intensity to highlight the nebulosities. The hard "blue" emission in the X-ray image and the optical image of the Homunculus show marked similarities in size, shape and orientation.

Figure 2 shows an overlay of the $3-8 \mathrm{keV}$ band X-ray contours on an HST image obtained by the Advanced Camera for Surveys (Ford et al. 2003) as part of the HST $\eta$ Carinae "Treasury Project"4. The isophots of the hard emission clearly follow the shape of the Homunculus, and there is very little hard X-ray emission beyond the Homunculus. Figure 3 shows the spatial variation of X-ray surface brightness through the Homunculus for 3 impact parameters, one through the central star and others between 3 and 4 arcseconds on either side of the star. The X-ray brightness is not uniform: the northwest lobe, and regions near the star are apparently brighter than the southeast lobe.

\footnotetext{
${ }^{4}$ http://etacar.umn.edu/
} 


\section{X-ray Spectroscopy of the Homunculus}

We extracted an X-ray spectrum of the Homunculus from an elliptical region of $22^{\prime \prime} \times 14^{\prime \prime}$ oriented with the semi-major axis along the polar axis of the Homunculus, excluding emission within 2.5 arcseconds of the star (which should exclude more than $95 \%$ of the direct stellar flux) and soft emission from the outer ejecta. The X-ray spectrum of the Homunculus is shown in Figure 4. This spectrum has been corrected for background emission, which was estimated from a source-free region to the northwest of the "red shell".

\subsection{The Thermal Component}

We created responses and effective areas for the ACIS observation, and fit the extracted spectrum with a combination of absorbed collisionally-ionized plasma models using the "mekal" model within the XSPEC analysis package (Arnaud 1996). For simplicity we assumed solar abundances although the composition of material around $\eta$ Carinae is decidedly non-solar. The X-ray emission associated with the Homunculus appears to be dominated by a component with a characteristic temperature of 113 million degrees, along with a weaker, cooler component ( $\sim 7$ million degrees). Table 1 compares the spectral properties (temperature, column density and luminosities) for the cooler and hotter components of the Homunculus, and the X-ray spectrum extracted from a $3.5^{\prime \prime}$ circle around the central star (corrected for background using the same background region as above).

The hard emission from the Homunculus is strongly absorbed, having an equivalent hydrogen column density $\left(N_{H}\right)=1.5 \times 10^{23}$ atoms $\mathrm{cm}^{-2}$, which is about the same as the absorbing column to the star. The absorbing column to the 6.7 million degree component is only $N_{H} \approx 2 \times 10^{21}$ atoms $\mathrm{cm}^{-2}$, which is consistent with foreground absorption. An examination of a low-energy X-ray image shows that this emission is clearly associated with the X-ray "bridge" (Weis et al. 2002; Weis, Corcoran, Bomans, \& Davidson 2004) running across the middle of the Homunculus.

\subsection{Fluorescent Emission}

The strongest feature in the X-ray spectrum of the Homunculus is an iron K-shell line at $6.4 \mathrm{keV}$, which we have modelled as a Gaussian in Figure 4. The inset in Figure 4 shows the ratio of the observed emission to a simple power law model which describes the continuum emission near the Fe line complex. The $6.4 \mathrm{keV}$ line is produced by fluorescent scattering of $\mathrm{X}$-rays by iron atoms (in either a gaseous phase inside the Homunculus, or locked in solid 
dust grains). The equivalent width of this iron line is about $1.5 \mathrm{keV}$, which is about a factor of 5 larger than the equivalent width of the iron fluorescent line seen in the spectrum of the star at times outside of the X-ray minimum. The line is significantly broadened $(\sigma \approx 0.1$ $\mathrm{keV}$ ) corresponding to a Doppler width of about $4700 \mathrm{~km} \mathrm{~s}^{-1}$. The fluorescent line in the stellar spectrum outside of minimum is consistent with $\sigma=0$ (Corcoran et al. 2001b). The velocity width of the line measured here is much larger than twice the measured expansion velocity of the Homunculus, which is only about $650 \mathrm{~km} \mathrm{~s}^{-1}$. The line broadening might be indicative of scattering off higher velocity flows in the lobes of the Homunculus. The velocity of $\eta$ Carinae's wind near the poles of the star has been measured to be $\approx 1100$ $\mathrm{km} \mathrm{s}^{-1}$ (Smith et al. 2003b), so that scattering from this material would produce a line broadening of $\lesssim 2200 \mathrm{~km} \mathrm{~s}^{-1}$, still a factor of 2 or more too low. At energies $>6.4 \mathrm{kev}$, some of the observed broadening is probably due to blending of narrow fluorescent $\mathrm{Fe}$ lines from $\mathrm{Fe}$ in a range of ionization stages. However, the fluorescent line shows an apparent "red wing" (as seen in the inset in figure 4) extending to $\sim 6.2 \mathrm{keV}$, which cannot be produced from line blending. Perhaps processes more exotic than simple Doppler broadening are in evidence. For example, Compton scattering can produce a "core" line with low energy "shoulders" (Matt 2002) though the intensity of the shoulder is expected to be much lower than the intensity of the core, which does not appear to be the case here (though it is conceivable that the line profile is produced by some superposition core lines + Compton shoulders given the complex scattering surfaces in the Homunculus). On the other hand, in systems like low-

mass X-ray binaries and active galaxies, the presence of a compact object can give rise to broad Fe K lines with significant red wings due to gravitational redshifts in accretion disks, but there is no strong evidence for the presence of a compact object in the $\eta$ Carinae system. It is perhaps most likely that the red wing is produced by X-ray scattering off neutral iron in the wind of $\eta$ Carinae's companion star, since the companion's wind velocity is believed to be $\sim 3000 \mathrm{~km} \mathrm{~s}^{-1}$ (Pittard \& Corcoran 2002), and the companion's wind should be receding from the observer at the time of the CHANDRA observation, based on the orbit presented in Corcoran et al. (2001). However, the companion's wind should be confined near the orbital plane, while the broad fluorescent line is apparently distributed throughout the entire Homunculus.

\section{Interpretation of the X-ray Emission from the Homunculus}

The high energy X-ray spectrum of the Homunculus is similar to the hard X-ray emission from $\eta$ Carinae itself in temperature and column density. Such emission could arise from shock-heated gas in the lobes of the Homunculus, but while shocked gas has been observed in (Smith 2002) and around (Dorland et al. 2003) the lobes, the deduced velocities are too 
low to produce significant emission at $8 \mathrm{keV}$. Furthermore, high velocity shocked gas could not exist in the lobes since such shocks are inconsistent with the presence of $\mathrm{H}_{2}$ which is seen in the lobes (Smith 2002). The high energy X-rays we observe from the Homunculus are instead an X-ray "light echo" in which hard X-ray photons produced in a strong, highvelocity shock near $\eta$ Carinae are reflected to the observer at earth, primarily by Thomson scattering off electrons in the Homunculus. Although the Homunculus contains significant amounts of dust, scattering from dust grains could not produce the Homunculus X-rays since dust scattering is so strongly forward peaked (Predehl \& Schmitt 1995).

The amount of scattering depends approximately on the ratio of the scattering cross section to the mass-weighted geometrical cross section of the lobes. For simplicity we consider the Homunculus as 2 hollow connected spheres with an axis running from the point of contact of the spheres through their centers, and assume that the axis is tilted by $45^{\circ}$ to the line of sight, with the northwest lobe tilted away from the observer. Assuming that the composition of the Homunculus is $50 \%$ helium and $50 \%$ hydrogen, the total number of electrons is roughly $N_{e}<5 \times 10^{57}$ and the total Thomson-scattering cross section is $A_{e}=N_{e} \times \sigma_{T}<3.6 \times 10^{33}$ $\mathrm{cm}^{2}$, where $\sigma_{T}$ is the Thomson scattering cross section $\left(0.665 \times 10^{-24} \mathrm{~cm}^{2}\right)$, assuming each lobe contains 6 solar masses (Smith et al. 2003a) as an upper limit. The approximate geometric radius of a lobe is very roughly $R_{L}=2.3 \times 10^{17} \mathrm{~cm}$ after weighting the lobe by the derived mass distribution (Davidson, et al. 2001). Thus the probability of scattering for an X-ray photon is roughly $P_{\text {scat }}<\left(A_{e} / 4 \pi R_{L}^{2}\right)=0.5 \%$. The observed scattering probability is roughly $P_{\text {scat }} \approx I_{H}(t) / I_{\eta}\left(t^{\prime}\right)$, where $I_{H}(t)$ is the intensity of the emission reflected from the Homunculus at the time of the CHANDRA observation, and $I_{\eta}\left(t^{\prime}\right)$ is the intensity of the star at an earlier time $t^{\prime}=t-\Delta t$, where $\Delta t$ is the light-travel time between the star and the reflecting surface, $\Delta t \approx R_{L} / c \approx 88$ days. Monitoring with the $R X T E$ observatory shows, 88 days prior to the CHANDRA observation (April 23, 2003, observation identification 8000101-54-00, Corcoran et al. 2004b, in preparation), that $I_{\eta}\left(t^{\prime}\right) \approx 1.6 \times 10^{35} \mathrm{ergs} \mathrm{s}^{-1}$, so that the observed scattering probability $P_{\text {scat }} \approx 3.4 \times 10^{31} / 1.6 \times 10^{35}=0.2 \%$, in very good agreement with the derivation given above for regions with appreciable light travel times.

\section{Spatial and Temporal Variations}

Regions of the Homunculus near the star where light travel times are negligible are also $\mathrm{X}$-ray bright. Figure 3 shows that the X-ray emission in the Homunculus on either side of the star is noticeably peaked near the star, though there is much less contrast in surface brightness on either side of the star. This suggests that, in the direction of the low-latitude reflecting surfaces, the stellar source may be substantially brighter than the stellar flux that 
we see directly, and/or the amount of scattering material in the region near the star may be much higher than the amount of material in the lobes. Another alternative is that there may be substantially more extinction to the X-ray emitting region along our line of sight than in the direction of these reflecting surfaces. In principal some or all of these suggestions may play a role. Detailed analysis of the variation of the surface brightness profiles depends on the time-varying flux from the central source, the real distribution of matter in the lobes, and the amount of material between the star and the reflecting surfaces.

Figure 5 shows a comparison between the ACIS S3 image obtained on August 28 and the HETG+ACIS S3 zeroth order image from the July 20 th observation. The images have been exposure corrected. The image on the left shows the August 28 image contours on the July 20th image. Both images are in the $3-8 \mathrm{keV}$ band. The shape and intensity of the emission from the Homunculus is very similar in the two observations. Figure 5 also shows a difference image in which the exposure corrected August 28 image is subtracted from the exposure corrected July 20 image. Contours in gray are negative contours (July 20 brighter than August 28) and black are positive. Though the brightness of the central source has increased substantially between the two observations, there is little change in intensity in the reflected emission from the Homunculus, although there is perhaps some evidence of a fading in the southeastern lobe.

\section{Conclusions}

We have presented the first clear detection of X-ray emission from the Homunculus nebula around $\eta$ Carinae, and have shown that this emission is consistent with the amount of stellar emission expected to be scattered from the Homunculus accounting for light-traveltime delays. Along with the scattered emission, we note the presence of strong $\mathrm{Fe} \mathrm{K} \mathrm{flu-}$ orescent line at $6.4 \mathrm{keV}$, which is anomalously broad, and which shows emission below 6.4 $\mathrm{keV}$.

The X-ray Homunculus is the nearest member of the small class of Galactic X-ray reflection nebulae (Murakami, Senda, Maeda, \& Koyama 2003), and the only one where both the emitting source and reflector are clearly identified. Variation in the intensity, orientation and spectral shape of the central source will be mirrored by variations in the scattered emission from the Homunculus, and in principle can help define the three-dimensional structure by providing view of the stellar X-ray source from multiple lines of sight, similar to the way in which reflected optical emission provides a latitudinally-dependent view of $\eta$ Carinae itself (Smith et al. 2003b). Continued monitoring of the reflected emission after the star's X-ray minimum would be especially interesting, since this could provide a measure of the intrinsic 
X-ray luminosity of the star during the minimum, and could help distinguish an occultation of the X-ray source from variation in the source's intrinsic X-ray brightness. Additional high spatial resolution observations of the X-ray echo from the Homunculus can help define the three-dimensional structure and variability of the stellar X-ray source. Observations of the reflected emission can also help map out the geometry of the absorbing material between the emitting region and the reflecting surface and allow us to more accurately model this unique system.

We gratefully acknowledge the exceptional support of Dr. Fred Seward and Dr. Norbert Schulz of the CHANDRA X-ray Center for their help in scheduling these observations. We also would like to thank the anonymous referee for helpful comments. This work was supported by SAO grant \#GO3-4008A. This research has made use of NASA's Astrophysics Data System. This research has made use of software obtained from the High Energy Astrophysics Science Archive Research Center (HEASARC), provided by NASA's Goddard Space Flight Center, software developed and provided by the CHANDRA X-ray Center, and the subpixel_resolution software developed by K. Mori at Penn State.

\section{REFERENCES}

Arnaud, K.A., 1996, Astronomical Data Analysis Software and Systems V, eds. Jacoby G. and Barnes J., p17, ASP Conf. Series volume 101.

Corcoran, M. F., Swank, J. H., Petre, R., Drake, S., \& Ishibashi, K. 1997, ASP Conf. Ser. 120: Luminous Blue Variables: Massive Stars in Transition, 267

Corcoran, M. F., Fredericks, A. C., Petre, R., Swank, J. H., \& Drake, S. A., 2000, ApJ, 545, 420

Corcoran, M. F., Swank, J. H., Petre, R., Ishibashi, K., Davidson, K., Townsley, L., Smith, R., White, S., Viotti, R., \& Damineli, A., 2001b, ApJ, 562, 1031

Corcoran, M. F., Ishibashi, K., Swank, J. H., \& Petre, R. 2001, ApJ, 547, 1034

Corcoran, M. F., 2003, IAUC 8160, 2

Damineli, A, 1996,ApJ, 460, L49

Damineli, A. 1997, ASP Conf. Ser. 120: Luminous Blue Variables: Massive Stars in Transition, 272 
Davidson, K., Smith, N., Gull, T. R., Ishibashi, K., \& Hillier, D. J., 2001, AJ, 121, 1569

Davidson, K. \& Humphreys, R. M. 1982, ARA\&A, 35, 1

Davidson, K., 1989, Ap\&SS, 157, 101

Davidson, K., et al., 2002, Space Telescope Science Newsletter ed. R. Brown, Spring, 1

Davidson, K., 1999, Astronomical Society of the Pacific Conf. Ser. 179, 304

Dorland, B. N., Currie, D. G., Kaufer, A., \& Bacciotti, F. 2003, Revista Mexicana de Astronomia y Astrofisica Conference Series, 15, 70

Ford, H. C., et al., 2003, Proc. of the SPIE 4854, 81

Garmire, G. P., Bautz, M. W., Ford, P. G., Nousek, J. A., \& Ricker, G. R., Jr., 2003, Proc. of the SPIE 4851, 28-44

Gaviola, E., 1950, ApJ, 111, 408

Hillier, D. John, Davidson, K., Ishibashi, K., \& Gull, T., 2001, ApJ, 553, 837

Ishibashi, K., Corcoran, M. F., Davidson, K., Swank, J. H., Petre, R., Drake, S. A., Damineli, A., \& White, S,1999,ApJ, 524, 983

Markert, T. H., Canizares, C. R., Dewey, D., McGuirk, M., Pak, C. S., \& Schattenburg, M. L., 1994, Proc. of the SPIE 2280, 168

Matt, G. 2002, MNRAS, 337, 147

Murakami, H., Senda, A., Maeda, Y., \& Koyama, K. 2003, ASP Conf. Ser. 289: The Proceedings of the IAU 8th Asian-Pacific Regional Meeting, Volume I, 175

Pittard, J. M. \& Corcoran, M. F. 2002, A\&A, 383, 636

Pittard, J. M., Stevens, I. R., Corcoran, M. F., \& Ishibashi, K., 1998, MNRAS, 299, L5

Predehl, P. \& Schmitt, J. H. M. M., A\&A, 293, 889

Seward, F. D., Forman, W. R., Giacconi, R., Griffiths, R. E., Harnden, F. R., Jones, C., \& Pye, J. P. 1979, ApJ, 234, L55

Seward, F. D., Butt, Y. M., Karovska, M., Prestwich, A., Schlegel, E. M., \& Corcoran, M. 2001, ApJ, 553, 832 
Smith, N., 2002, MNRAS, 337, 1252

Smith, N., Gehrz, R. D., \& Krautter, J. 1998, AJ, 116, 1332

Smith, N., Gehrz, R. D., Hinz, P. M., Hoffmann, W. F., Hora, J. L., Mamajek, E. E., \& Meyer, M. R., 2003a, AJ, 125, 1458

Smith, N., Davidson, K., Gull, T. R., Ishibashi, K., \& Hillier, D. J., 2003b, ApJ, 586, 432

Smith, N., et al., 2004, ApJ, 605, 405

Tsunemi, H., Mori, K., Miyata, E., Baluta, C., Burrows, D. N., Garmire, G. P.; \& Chartas, G. 2001, ApJ, 554, 496

Weis, K., Corcoran, M. F., \& Davidson, K., 2002,ASP Conf. Ser., 262, 275

Weis, K., Corcoran, M. F., Bomans, D. J., \& Davidson, K. 2004, A\&A, 415, 595

Weisskopf, M. C., Tananbaum, H. D., Van Speybroeck, L. P., \& O'Dell, S. L., 2000, Proc. of the SPIE 4012,2

Weisskopf, M. C., O'dell, S. L., \& van Speybroeck, L. P., 1996, Proc.of the SPIE 2805, 2 


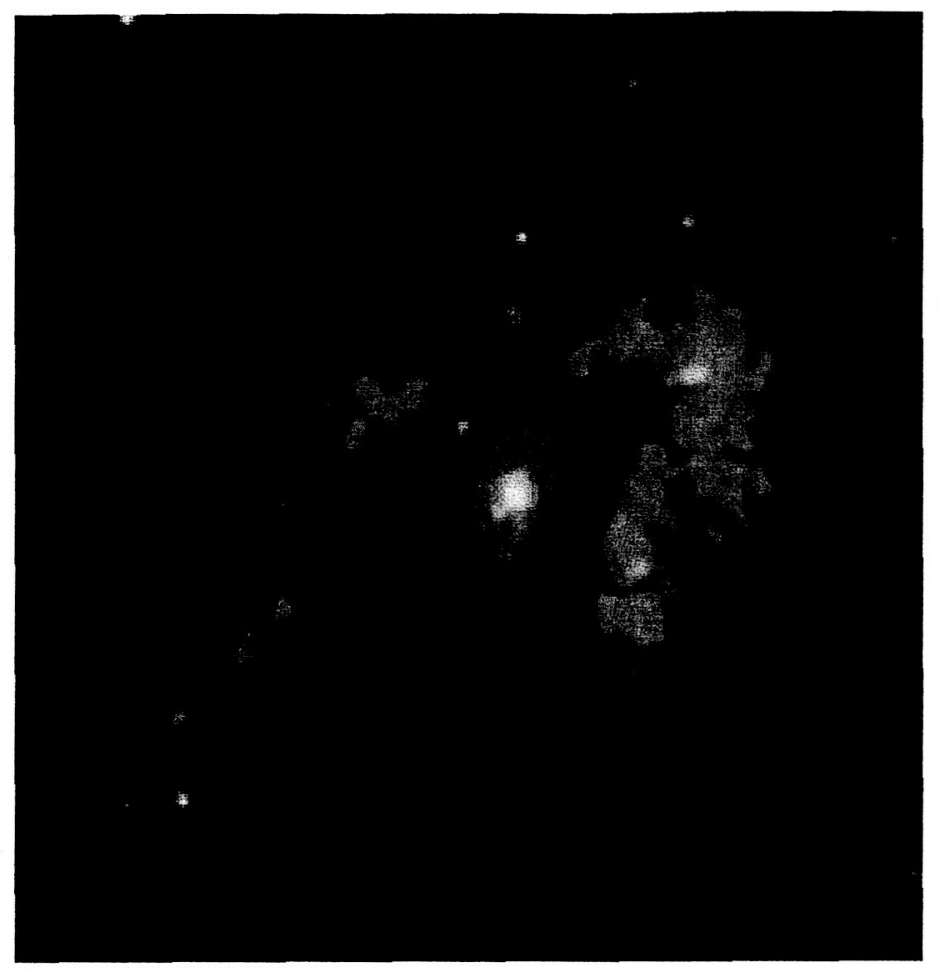

Fig. 1.- Left: A color X-ray image of $\eta$ Carinae during the star's most recent X-ray minimum obtained by the CHANDRA X-ray observatory. North is to the top, and East to the left. Red indicates low-energy X-ray emission $(0.2-1.5 \mathrm{keV})$, green medium energy $(1.5-3.0 \mathrm{keV})$ and blue high-energy emission $(3.0-8.0 \mathrm{keV})$. Emission from the star itself is visible as a white point source at the center of the image. A red, broken elliptical ring of emission lies beyond the star. The bluish patch around the star inside this ring is reflected X-ray emission from the Homunculus Nebula. Right: An HST/WFPC2 [NII] $\lambda 6583$ image of $\eta$ Carinae. The plate scale and orientation of the Hubble and CHANDRA images are the same. 


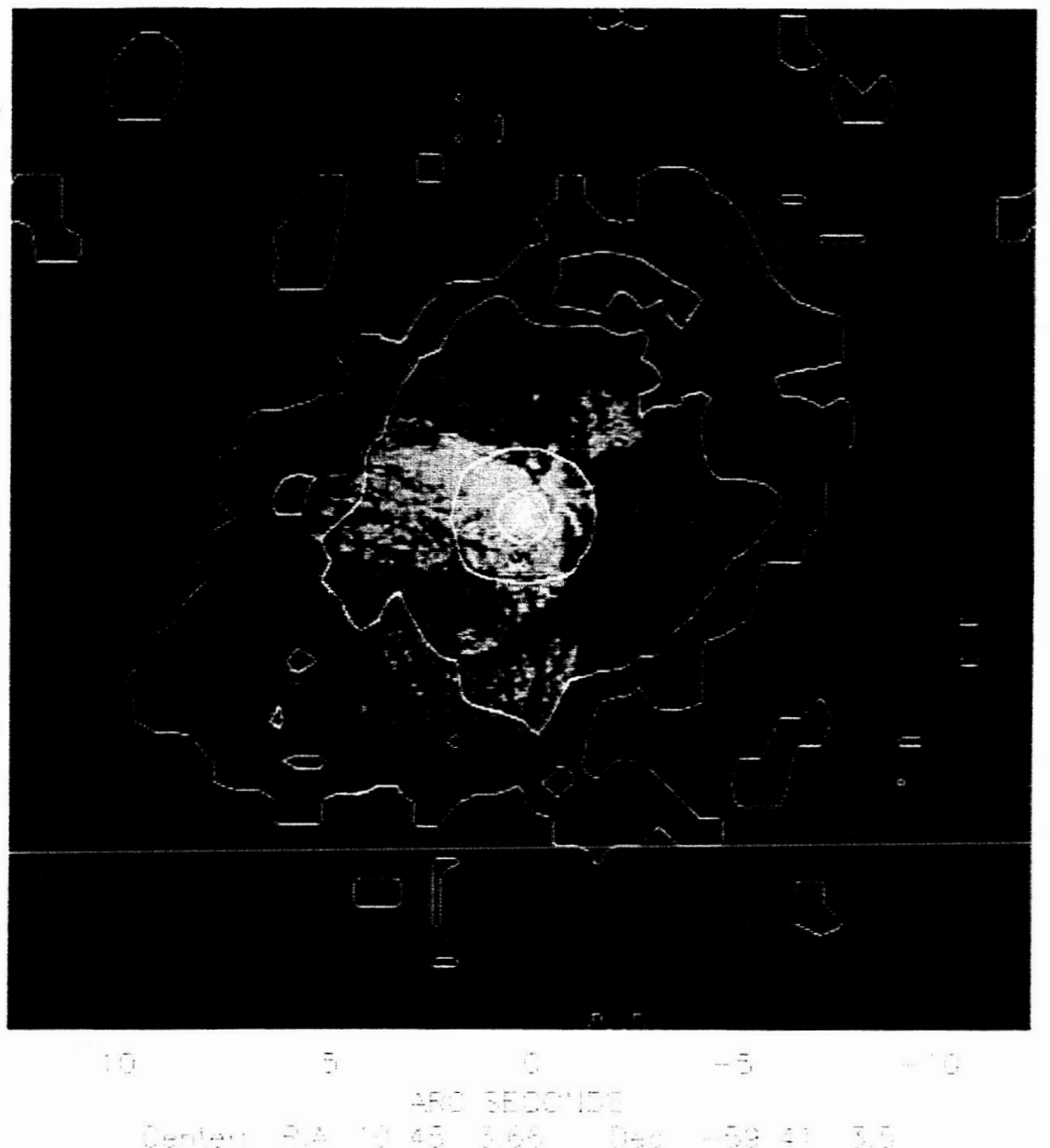

Fig. 2.- Contours of the 3-8 keV X-ray emission on a Hubble ACS image of the Homunculus. Contour levels are $0.1,0.7,6.9$ and $68.9 \%$ of the peak brightness. 


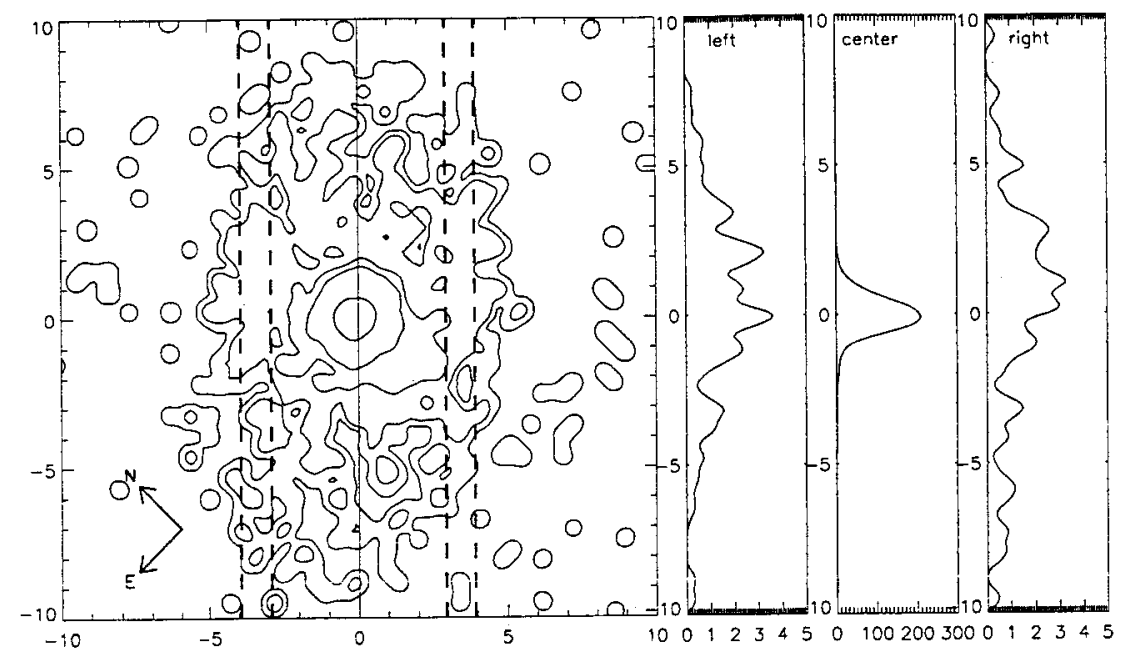

Fig. 3.- The image on the left is the contour map of the 3-8 keV X-ray surface brightness of the Homunculus. Surface brightness profiles $10^{\prime \prime}$ to the left of the star, on the star, and $10^{\prime \prime}$ to the right of the star are shown next to the contoured image. The location of the surface brightness cuts on either side of the star is shown by the vertical dashed lines. The left and right brightness profiles are integrated between the dashed lines. 


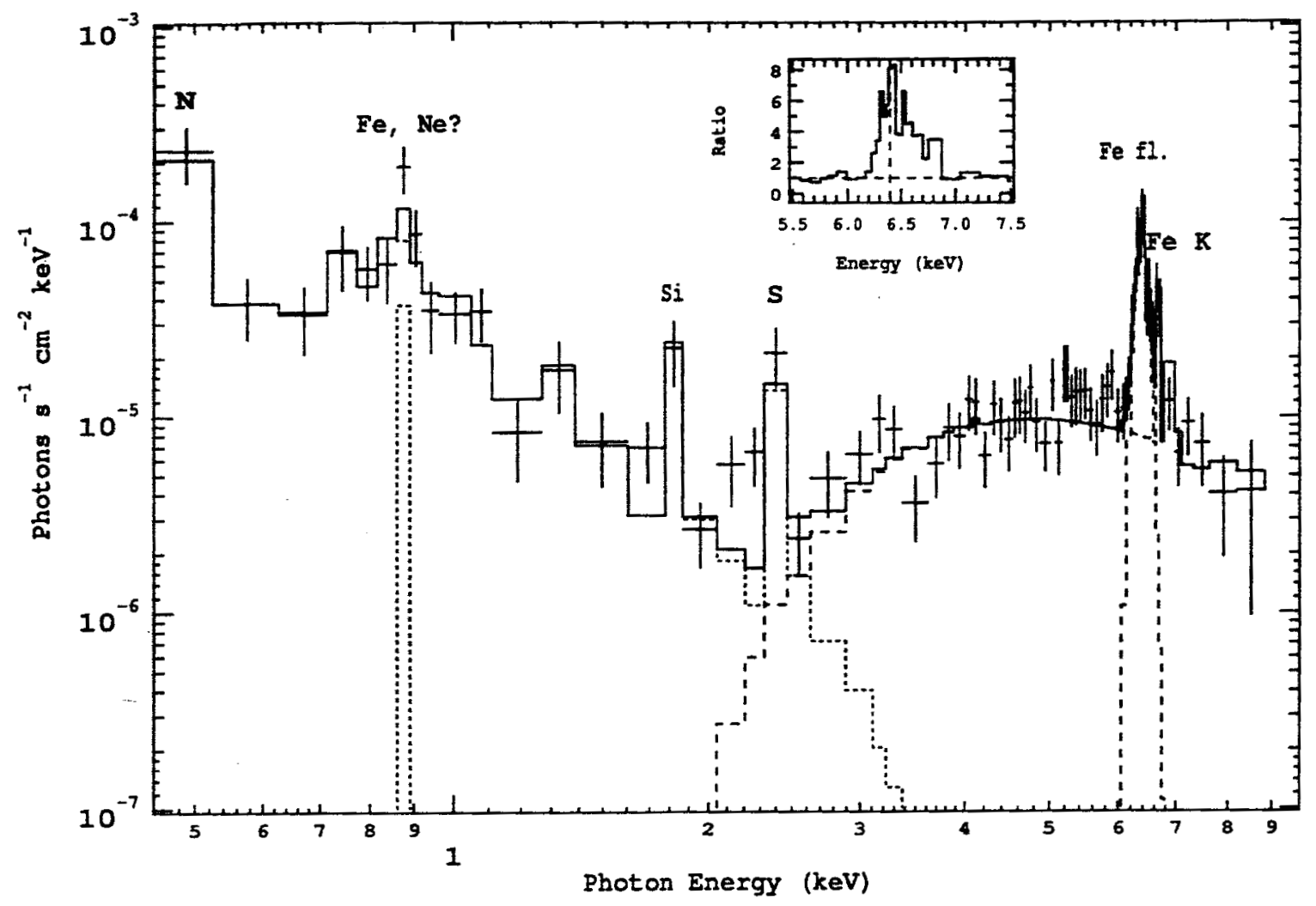

Fig. 4.- The X-ray spectrum of the Homunculus from the July 20th observation. The model spectrum is shown as an unbroken line, with individual components shown by broken lines. An emission line near $0.9 \mathrm{keV}$ may be emission from Fe XVII or Ne IX, while the strongest emission line at $6.4 \mathrm{keV}$ is produced by fluorescent scattering of X-rays by iron atoms in the Homunculus. Emission from at least two plasmas, one at a temperature near 7 million degrees and one at a temperature of about 100 million degrees are required to describe the observed spectrum. The inset shows the ratio of the emission near the Fe line complex at 6.4-6.8 keV to a simple power law model to describe the continuum emission. Significant emission below $6.4 \mathrm{keV}$ is observed in the $\mathrm{Fe} \mathrm{K}$ fluorescent line. 

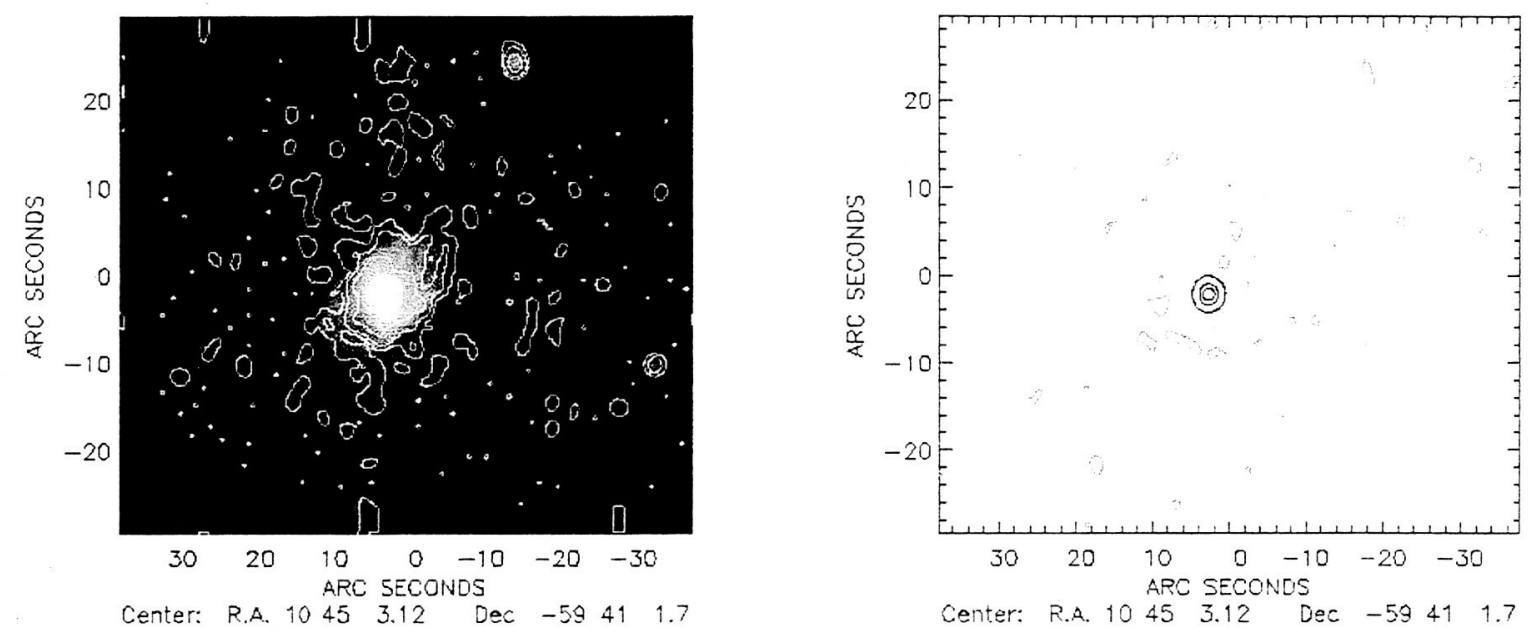

Fig. 5.- The image on the left shows contours of the August 282003 observation on the July 20th ACIS zeroth-order image. The image on the right shows a contour image of the exposure corrected August 28 image minus the exposure-corrected July 20 image. Negative contours are shown in gray. The central source is significantly brighter in August 28 compared to the earlier observation. 
Table 1: Spectral Characteristics of the Emission from the Homunculus

\begin{tabular}{lcccc}
\hline \hline Component & $\begin{array}{c}\text { Temperature } \\
\left(10^{6} \mathrm{~K}\right)\end{array}$ & $\begin{array}{c}\text { Column Density } \\
\left(10^{22} \mathrm{~cm}^{-2}\right)\end{array}$ & $\begin{array}{c}L_{x}^{\mathrm{a}} \\
\left(10^{31} \mathrm{ergs} \mathrm{s}^{-1}\right)\end{array}$ & $\begin{array}{c}L_{x, c} \mathrm{~b} \\
\left(10^{31} \mathrm{ergs} \mathrm{s}^{-1}\right)\end{array}$ \\
\hline Homunculus (soft) & $6.7 \pm 2.7$ & $0.2 \pm 0.1$ & $17.7 \pm 2.1$ & 19.8 \\
Homunculus (hard) & $113 \pm 66$ & $15 \pm 3.3$ & $46.6 \pm 4.3$ & 113 \\
star & $58.0 \pm 3.5$ & $15 \pm 4.5$ & $142.4 \pm 5.1$ & 311.4 \\
\hline
\end{tabular}

a Observed luminosity in the $0.5-10 \mathrm{keV}$ band, assuming the distance to $\eta$ Carinae is $2300 \mathrm{pc}$ (Hillier et al. 2001).

${ }^{b}$ Absorption corrected luminosity in the $2-10 \mathrm{keV}$ band 